\title{
No easy solutions for aerospace
}

\author{
The application of advanced materials in aerospace presents multiple scientific and regulatory \\ challenges that must be addressed.
}

Civil aviation is known to be a significant contributor to global warming, with highaltitude $\mathrm{CO}_{2}$ and condensation trails being important factors ${ }^{1}$. With the number of passengers ever increasing - China for example is forecast to experience an average year-on-year growth rate of 5.5\% (ref. 2), the largest of any country - this contribution is likely to rise. As for many modes of transportation, reducing weight and enhancing efficiency are key goals. For example, the use of lightweight magnesium alloys for aircraft seats, previously limited by concerns over the flammability of magnesium, was permitted in $2015^{3}$. Gasturbine engines are a mainstay of the aviation industry and, therefore, reducing their weight by moving towards lower-density alloys, and improving combustion efficiency by higher operating temperatures is critical. In this focus issue of Nature Materials we explore some of the key challenges that face the aerospace community, with particular spotlights on high-temperature materials for gas-turbine engines (Fig. 1), and barriers to the uptake of new material-based technologies.

Developing materials for jet engines is extremely tricky. High temperatures open up a sizeable palette of structure-altering phenomena, exacerbated by substantial mechanical loads. The intricacy of the problem is further amplified by complex load regimes during the course of a flight, and by the rotation of turbine components. Resistance to chemical attack is also important. These conditions place unprecedented demands on materials. In an interview on page 819 David Rugg, Senior Engineering Fellow in Materials at Rolls-Royce plc., discusses further complications: being able to predict properties and structure is necessary to assure in-service reliability and safety. As he mentions, understanding the basic behaviour of materials is needed to establish accurate predictive models - experimental characterization under multiple conditions is not enough - and collaboration between industry and academia is required to achieve this.

A wide range of material classes can help to meet the demands of aerospace. High-strength, lightweight polymer-based composites are attractive for the fuselage and wings, while metals and ceramics are utilized in jet engines, due to their potential for high operating temperatures. In this regard, complex material systems including nickelbased superalloys for turbine blades and disks, with ceramic-based thermal barrier coatings to further increase the allowable temperature, are vital. On pages 804 and 809 Nitin Padture and Tresa Pollock separately discuss the use of ceramics and high-temperature alloys, respectively, for propulsion in aerospace. Titanium aluminides in particular are being sought as a replacement for Ni-based superalloys in some parts of the jet engine, since they offer weight savings, and are now

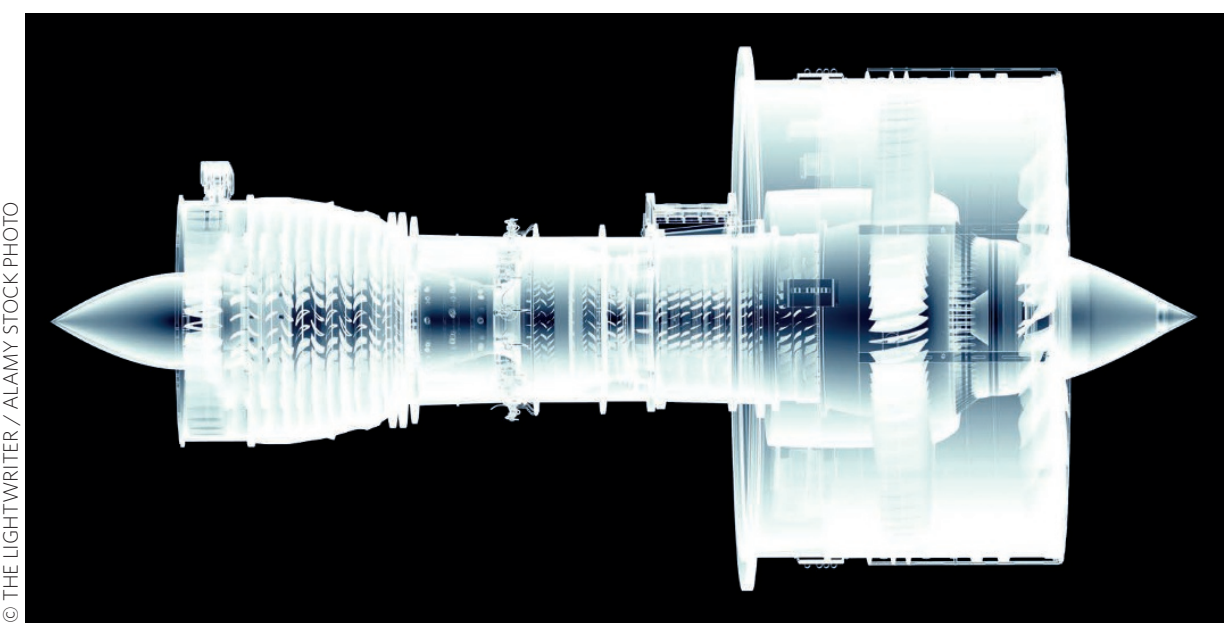

Figure 1 | Jet engines expose materials to high temperatures, cyclic mechanical loads and corrosive environments. Knowing how materials respond to these variable conditions is vital to their reliability.

being used in General Electric's engines for Boeing's 787 Dreamliner $^{4}$ (which also featured an impressive $50 \%$ by weight of composites $^{5}$ ). On page 876 Chen et al. report a new seedless crystal-growth method for titanium aluminides, allowing them to obtain a combination of high-temperature strength, ductility and creep resistance.

Underpinning scientific challenges for the introduction of new technologies in aerospace are strict safety requirements that must not be compromised. These present substantial issues for nurturing a new technology. On page 815 Roca et al. discuss the need for US government input to enable metal additive manufacturing (MAM) to bridge the 'valley of death' from nascent technology to wider uptake in the aerospace industry. They mention that more should be done to establish flexible regulations to guide the MAM community in developing the technology, and that applications that are not safety-critical should be sought. This second recommendation is particularly relevant to aerospace technologies: the priority of safety means that new materials and designs must stringently prove themselves before application, yet it is only by their usage that they can be truly understood. This 'catch-22' situation requires easier routes for new technologies to find their way into services where safety is not so critical, where they can be further tested and developed.

It is clear that the application of new materials and technologies in aerospace presents scientific, engineering and regulatory issues. That safety is rightly placed as a cornerstone of this industry creates sizeable challenges. However, given the importance of aerospace materials in reducing global warming effects, and the considerable economic rewards that drive this industry, there is no doubt that challenges will be addressed despite a simple solution often being elusive.

\footnotetext{
References

1. Boucher, O. Nature Clim. Change 1, 24-25 (2011).

2. New IATA passenger forecast reveals fast-growing markets of the future. IATA (16 October 2014)

3. Magnesium Elektron welcomes new standard on use of magnesium in commercial aircraft seats. Magnesium Elektron (21 September 2015)

4. Clark, S. F. 787 Propulsion System (Boeing, 2012); http://go.nature.com/29oA2VJ

5. Hale, J. Boeing 787 From the Ground Up (Boeing, 2006); http://go.nature.com/29kgufs
} 